\title{
EMISSION LINE VARIATIONS OF BLRG
}

\author{
M. DIETRICH and W. KOLLATSCHNY \\ Universitätssternwarte, Geismarlandstr. 11, D-37083 Göttingen, F.R.G.
}

In late 1989 we started a monitoring campaign of the line profile variations of more than 40 Broad-Line Radio Galaxies (BLRG) at Calar Alto Observatory/Spain. BLRG are the most extreme species of AGN regarding line width and structure of their optical emission line profiles showing FWZI up to $35000 \mathrm{~km} \mathrm{~s}^{-1}$, eg. $3 \mathrm{C} 332$ or Arp102B. Quite often the broad emission line profiles are characterized by a double hump structure. The analysis of the broad emission lines provides information about fundamental parameters of the inner part of the AGN like size, structure and kinematics of the line emitting region. In the following we present line profile variations of a BLRG we are studying.

Spectra of 3C390.3 at different epochs are displayed in Fig. 1 together with the corresponding difference spectrum. Prominent double peaked profiles of the $\mathrm{H} \alpha$ and $\mathrm{H} \beta$ emission lines are visible in the difference spectrum. These line profiles were transformed to the velocity space (cf. Fig. 1). In both lines the humps are shifted by $v_{\text {rel }}=-2000 \mathrm{~km} \mathrm{~s}^{-1}$ and $v_{\text {rel }}=+7000 \mathrm{~km} \mathrm{~s}^{-1}$, respectively.

This work has been supported by DFG grant Ko 857/13-1
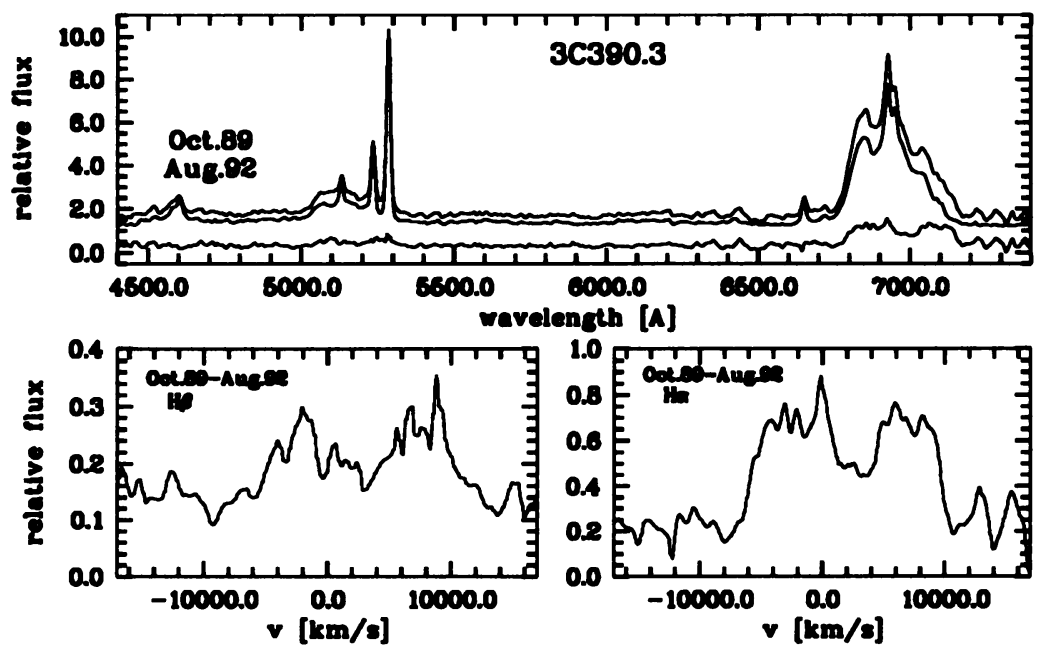

Fig. 1. Spectra of 3C390.3 taken at Aug. 31, 1992 and Oct. 28, 1989. The difference spectrum is also shown. The line profiles of $\mathrm{H} \beta$ and $\mathrm{H} \alpha$ of the difference spectrum were transformed to the velocity space and are displayed above

T. J.-L. Courvoisier and A. Blecha: Multi-Wavelength Continuum Emission of AGN, 444.

(C) 1994 IAU. Printed in the Netherlands. 